\title{
Development of die-bonding film by nano-structure control and mathematical optimization
}

\author{
Teiichi Inada
}

Recent advances in electronic equipment demand more compact, lighter and functionally superior components. Die-bonding film is widely used in semiconductor packaging in electronic equipment such as smart phones and personal computers. The requirements for the die-bonding film used in such devices include high adhesive strength and heat resistance. To meet the requirements mentioned above, an acrylic polymer-matrix epoxy adhesive, which was based on reaction-induced phase decomposition, was selected. Die-bonding film based on these polymer-matrix systems shows higher flexibility under thermal stress during the heat cycle test than film based on epoxy-matrix systems. In addition, the properties of the polymer-matrixbased films vary widely depending on the ratio of epoxy resin and acrylic polymer contents. To satisfy the target properties, a novel material design method named weak conditioned combinatorial linear programming was developed. Optimization via this method can be performed using the newly developed user-friendly software.

Polymer Journal (2014) 46, 745-750; doi:10.1038/pj.2014.66; published online 20 August 2014

\section{INTRODUCTION}

State-of-the-art electronic devices such as smart phones (Figure 1), tablets, ultra-thin laptops and servers that support cloud storage are evolving continuously. Semiconductor packaging, as shown in Figure 1, is at the heart of such devices and is also evolving rapidly. Various materials are used in semiconductors. In recent years, multilayered semiconductor packaging has been one of the most important technologies that enhance the performance of electronic devices. To satisfy the intricate requirements for electronic devices, the mounting density of semiconductors per unit area has to be increased, requiring new packaging methods that use high-performance materials. To bond each layer of a multi-chip package, die-bonding film is widely used. For example, as shown in Figure 2, several types of diebonding film are used in semiconductor packaging; thus, the requirements for the film vary depending on the application. Processes for manufacturing stacked packages and the requirements for die-bonding film are shown in Figure 3. Assembly processes for semiconductor packaging are also diverse, and various properties are required for each process. Commonly required properties include the following:

(1) The film must be tacky within a temperature range of $60-80^{\circ} \mathrm{C}$, and it must be able to adhere to the wafer.

(2) Because the film is used to bond the chip to the substrate, it must be able to deform to accommodate differences in the coefficient of thermal expansion to relieve stress.

(3) The film must not flow or detach during the soldering process (approximately $260^{\circ} \mathrm{C}$ ) that is used to attach the semiconductor package to the wiring substrate.
The reaction-induced phase decomposition system with acrylic polymer and epoxy resin, which is flexible, heat resistant and easy to design, has properties that can be applied to such applications and processes.

\section{RESULTS AND DISCUSSION}

Material system based on reaction-induced phase decomposition In electronic devices, epoxy resin has been widely used to achieve various high-performance functionalities such as high heat resistance; however, epoxy resin is too hard to be applied to stacked packages in which many different types of material are laminated. To develop the die-bonding film, the reaction-induced phase decomposition ${ }^{1,2}$ of an acrylic polymer/epoxy system was selected. The film consists of copolymerized acrylic polymer, which is a random copolymer of acrylonitrile and methacryl and acryl ethers, epoxy resin (bifunctional and multifunctional epoxy resin), curing agent, catalyst and inorganic fillers. In this system, the acrylic polymer and epoxy resin phases separate after curing (Figure 4), although the acrylic polymer and epoxy resin are miscible with each other before curing. ${ }^{3}$ This polymer-matrix system shows a higher degree of flexibility under thermal stress during the heat cycle than do epoxy-matrix systems. ${ }^{4-6}$

The final morphology of the cured system depends on competition between the cross-linking reaction and the phase decomposition during curing. Therefore, controlling these two processes by varying the polymer/epoxy ratio, type and amount of curing agent added and by optimizing the curing conditions is essential for achieving the desired phase morphology. The modulus of the film is widely controlled by varying the polymer/epoxy ratio and the amount of filler (Figure 5). ${ }^{4}$ 
The die-bonding film (the ratio of acrylic polymer/epoxy resin/ hardener (diaminodiphenylmethane) is $70 / 23 / 7$ ) was laminated on the polyimide (PI) film and cured at $120^{\circ} \mathrm{C}$ for $1 \mathrm{~h}$. The epoxyresin phase of the film deposited onto the PI interface gives a tilted (compositional gradient) structure as shown in Figure $6 .^{7}$ The epoxy resin domain is localized near the PI film interface, and it improves the adhesion strength. The phase separation structure at

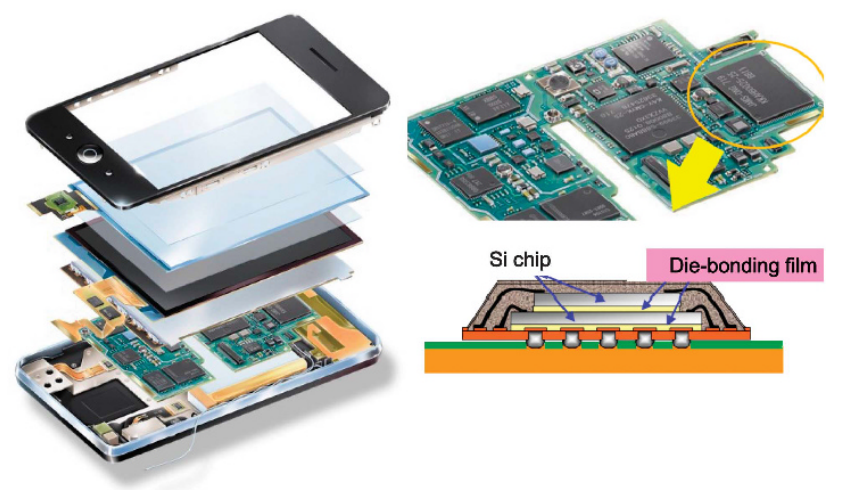

Figure 1 Electronic device and structure of semiconductor packaging.

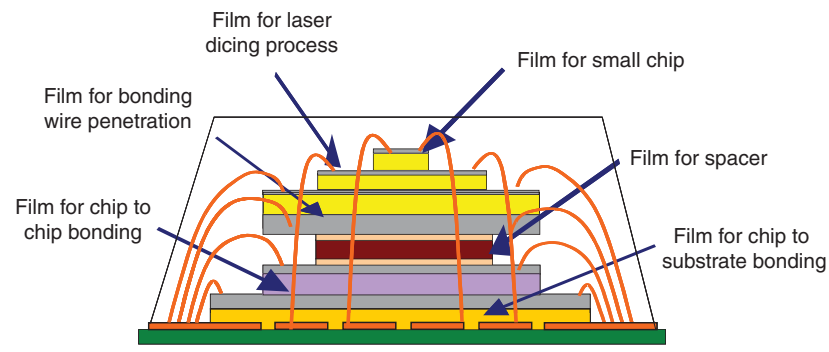

Figure 2 Application of die-bonding film for several stacking structures in semiconductor packaging. the nanoscale is also formed in the sea phase of the acrylic polymer, forming islands of epoxy resin. After the T-peel test of the sandwich film composed of PIs and die-bonding film, cavitation (cavernous phenomenon) was observed near the stripped plane of the sandwich film composed of PI and die-bonding film, as shown in Figure 7.

Novel die-bonding film was developed using reaction-induced polymer alloy technology, precise film manufacturing and technology for evaluation of semiconductor packaging. Prior to curing, the film showed excellent adhesion strength, reliability and heat resistance, and it satisfied the demands of the semiconductor package assembly process.

\section{Material design system}

Die-bonding film is a composite material made by combining various materials, and a variety of properties can be obtained by changing the composition ratio. However, the optimization of all properties is difficult because all properties have a trade-off relationship (Figure 8). To solve this issue, a novel design method based on linear programming ${ }^{8}$ (shown in the equation below) was constructed.

$$
\left(\begin{array}{c}
p_{1} \\
p_{2} \\
\vdots \\
\vdots \\
p_{m}
\end{array}\right)=\left(\begin{array}{ccc}
a_{11} & \cdots & a_{1 n} \\
a_{21} & & a_{2 n} \\
\vdots & \ddots & \vdots \\
\vdots & & \vdots \\
a_{m 1} & \cdots & a_{m n}
\end{array}\right)\left(\left(\begin{array}{c}
k_{1} \\
k_{2} \\
\vdots \\
\vdots \\
k_{n}
\end{array}\right) \otimes\left(\begin{array}{c}
c_{1} \\
c_{2} \\
\vdots \\
\vdots \\
c_{n}
\end{array}\right)\right)
$$

The matrix consisting of $a_{m n}$ is the property/composition matrix, For example, $a_{m n}$ shows the relationship between the composition content and the adhesiveness of an epoxy resin. $\left(\begin{array}{lll}c_{1} & \ldots & c_{n}\end{array}\right)$ is the content vector, $\left(p_{1} \ldots p_{n}\right)$ is the property vector, $\left(\begin{array}{llll}k_{1} & \ldots & k_{n}\end{array}\right)$ is the combination vector and $\otimes$ is the Hadamard product as

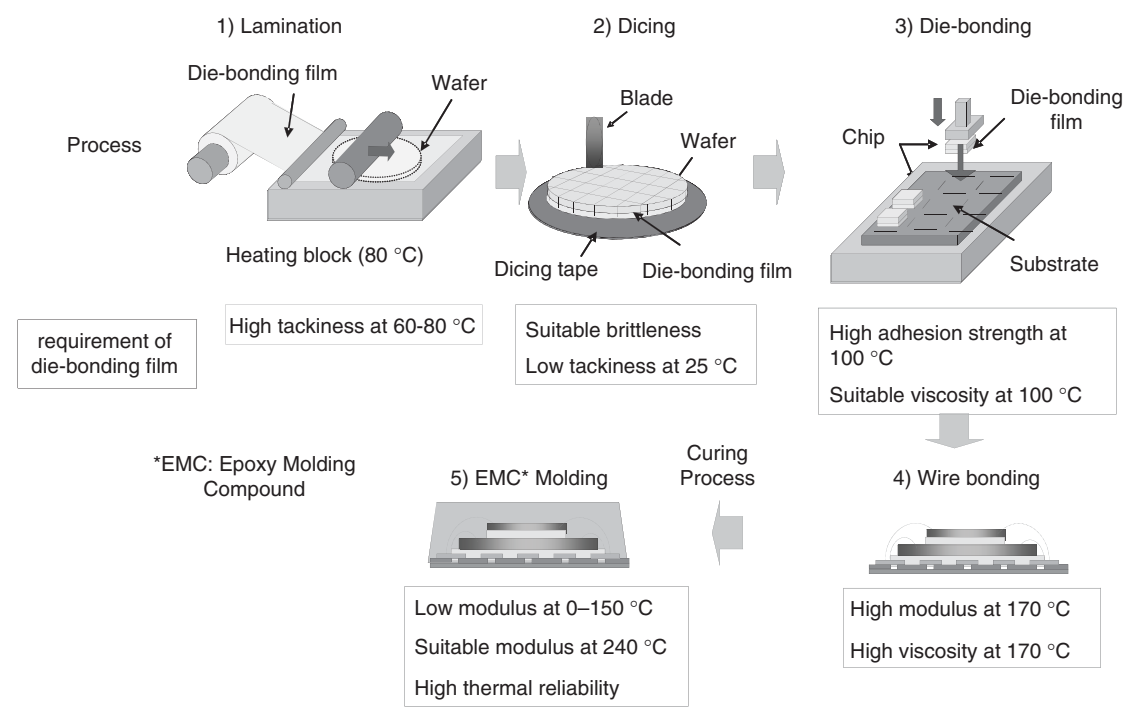

Figure 3 Manufacturing process of stacked packaging and requirements of die-bonding film. A full color version of this figure is available at Polymer Journal online. 
a

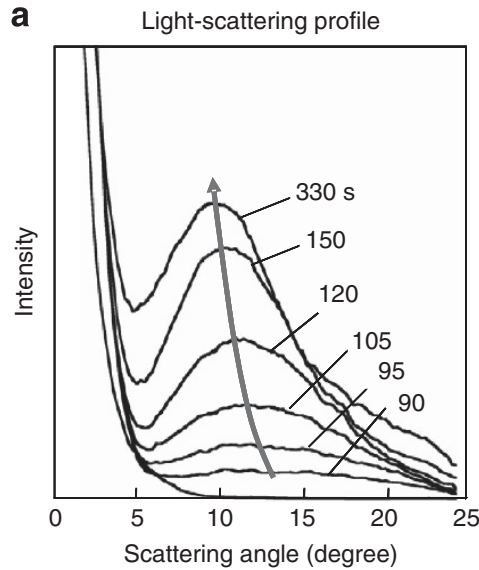

b

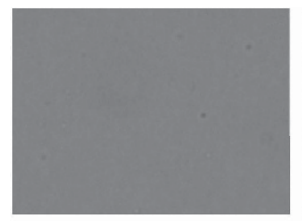

$105 \mathrm{~s}$

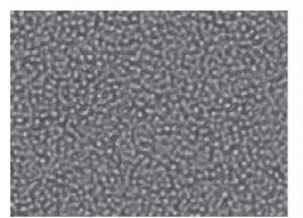

$150 \mathrm{~s}$
Phase-contrast micrograph

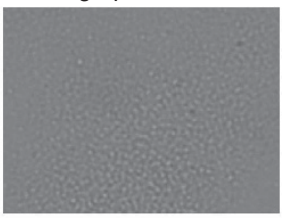

$120 \mathrm{~s}$

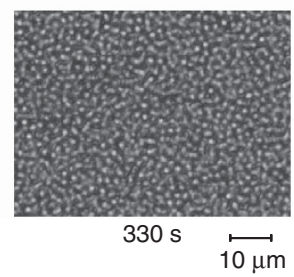

Figure 4 Change in the light scattering profile (a) and phase-contrast micrograph (b) with curing at $170{ }^{\circ} \mathrm{C}$. A full color version of this figure is available at Polymer Journal online.

defined below.

$$
\begin{aligned}
\boldsymbol{A} \otimes \boldsymbol{B} & =\left(\begin{array}{ccc}
a_{11} & \cdots & a_{1 n} \\
\vdots & \ddots & \vdots \\
\vdots & & \vdots \\
a_{m 1} & \cdots & a_{m n}
\end{array}\right) \otimes\left(\begin{array}{ccc}
b_{11} & \cdots & b_{1 n} \\
\vdots & \ddots & \vdots \\
\vdots & & \vdots \\
b_{m 1} & \cdots & b_{m n}
\end{array}\right) \\
& =\left(\begin{array}{ccc}
a_{11} \cdot b_{11} & \cdots & a_{1 n} \cdot b_{1 n} \\
\vdots & \ddots & \vdots \\
\vdots & & \vdots \\
a_{m 1} \cdot b_{m 1} & \cdots & a_{m n} \cdot b_{m n}
\end{array}\right)
\end{aligned}
$$

The material combination $k_{n}$ parameter of material $n$ is set as 1 when it is used and 0 when it is not used.

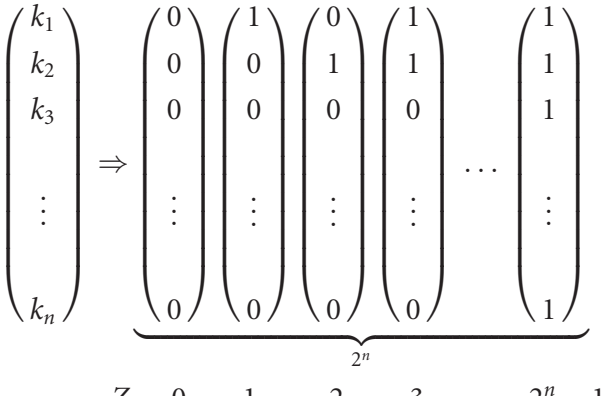

$$
\begin{aligned}
& \begin{array}{llllll}
Z=0 & 1 & 2 & 3 & \ldots & 2^{n}-1
\end{array}
\end{aligned}
$$

The material combination vector will be in one-to-one correspondence with the combination index (described as $Z$ ). For example, when considering the composition by selecting $0-n$ type of materials from $n$ number of materials, there will be $2^{n}$ combinations. If the expression of a combination is expressed as $010101 \ldots$, the expression becomes complicated when there are many type of materials. Therefore, the number sequence is set as binary, and one-to-one correspondence is given to decimal $Z$. The corresponding composition parameter $c_{n}$ is changed to determine whether the property values lie within the target range, and this will determine whether the composition range satisfies the target value.

While conventional linear programming sought the maximum or minimum values of the evaluation function, all the solutions that satisfy the target values are considered as the solution set. Therefore,

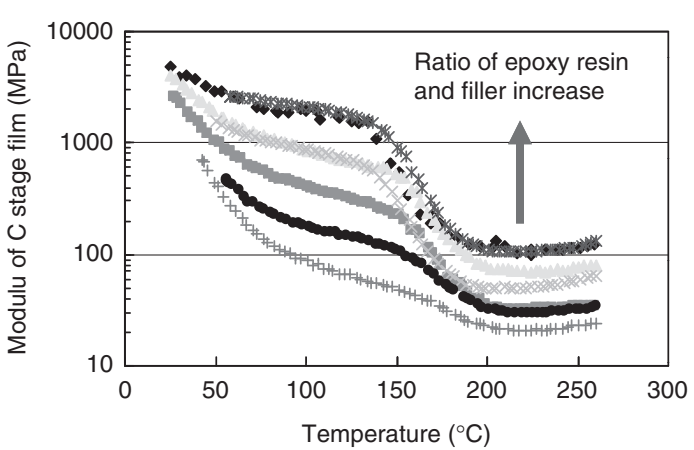

Figure 5 Storage modulus of polymer alloy films with varying amounts of acrylic polymer and filler. A full color version of this figure is available at Polymer Journal online.

this is called a 'weak condition.' Weakening the condition of the solution helps to shorten the computation time. ${ }^{9-11}$

Mathematically, as shown in Figure 9, the weak conditioned combinatorial linear programming creates space $P$ of realizable properties, as space $C$ (created by the composition parameter $c_{n}$, which possesses limitations) is linearly mapped to the property space by the matrix consisting of the composition/property parameter. The product set $S(=P \cap T)$ of space $P$ and space $T$ of the target value comprise the space that satisfies the target value. Additionally, space $C_{S}$ is created by the compositional parameter, $c_{n}$, that satisfies $S$, and this is the composition that satisfies the target property. The wider the range of $S$ or $C_{S}$, the more capable it will be to address changes in the required property in the future, and such breadth is preferable. In practice, the cost and tolerance are also considered within this composition to determine the optimal final composition.

The aforementioned mathematical process is too complex for a material engineer to conduct as a daily routine, and the volume of computation becomes extremely large when $n$ increases because it is necessary to calculate the solution space for every combination. It is nearly impossible to calculate the solution space using Excel or manually. Therefore, the solution search software M-Designer, ${ }^{9-11}$ which can be used practically for such a purpose, was developed. M-Designer is a software program with an interface, as shown in Figure 10. Using M-Designer, it is possible to compute whether there is a composition (solution set) that satisfies the target value for each 


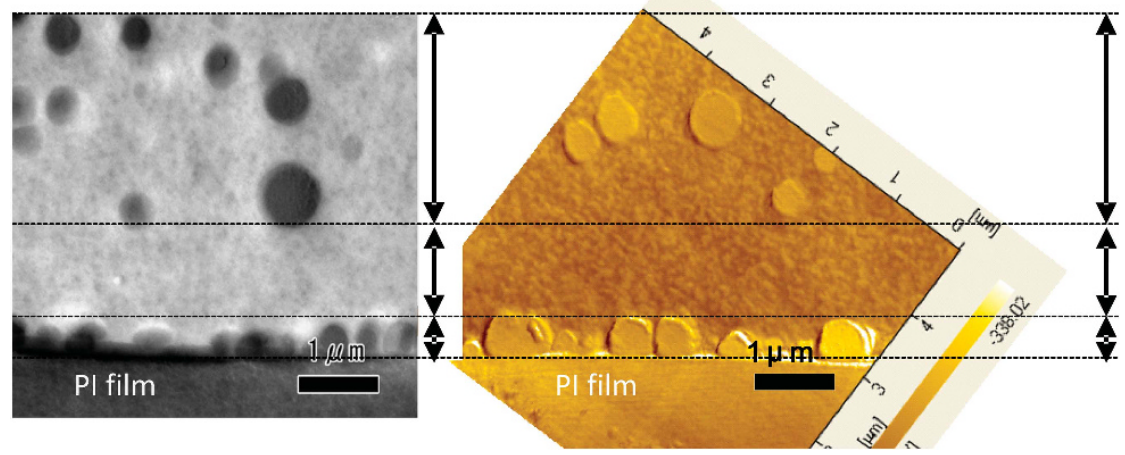

Figure 6 Transmission electron microscopy and scanning viscoelasticity microscopy image of the adhesive film interface.

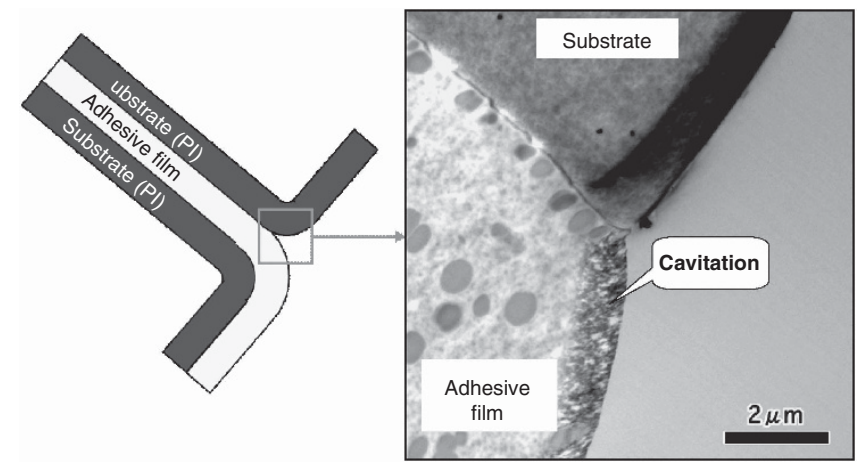

Figure 7 Transmission electron microscopy image of the debonding process. A full color version of this figure is available at Polymer Journal online.

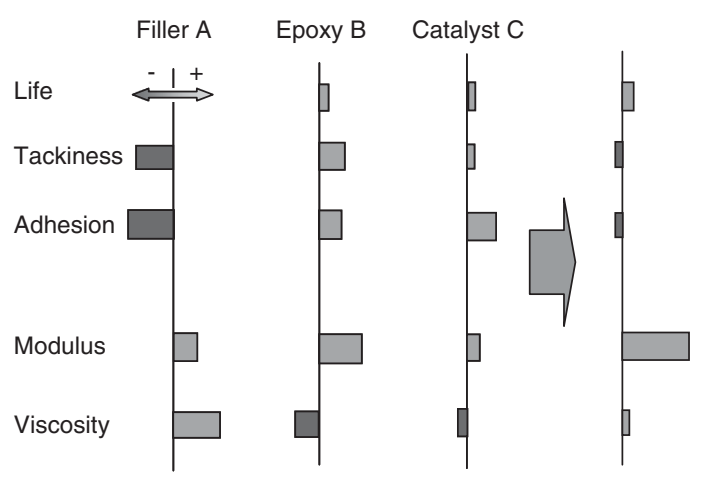

Figure 8 Example of the trade-off relationship of chemical formulations. A full color version of this figure is available at Polymer Journal online.

combinatorial index. Specifically, the maximum and minimum values of the property/composition matrix, the range of the target property, the number of compositions and the content (obtained experimentally or theoretically) are entered. The content of the material is automatically changed at appropriate intervals, and the decision is made automatically as to whether the material composition satisfies the target. The compositions that satisfy the target value are then provided.

The composition candidates must simply satisfy the target value; it is not necessary to seek the optimal value, but rather the acceptable range. If the range is wide, the possibilities of various usages increase.

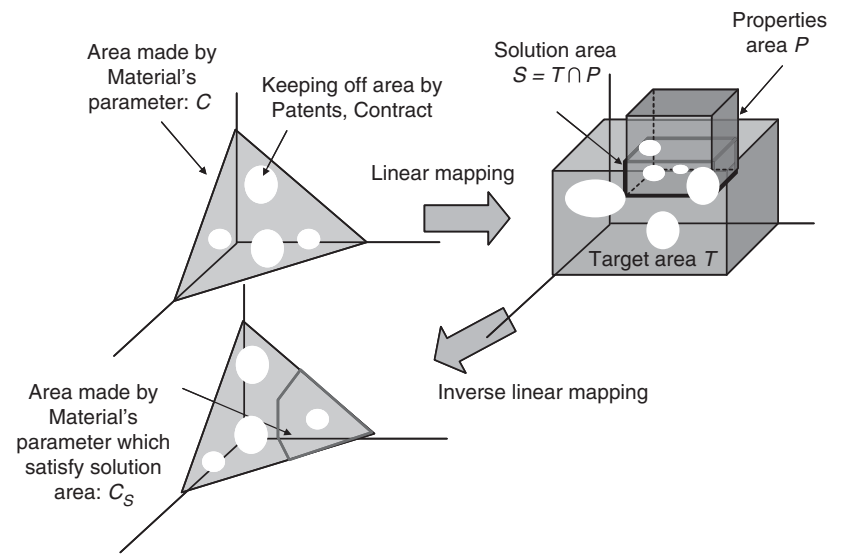

Figure 9 General representation of the solution area and mapping defined by a matrix that shows the correlation between properties and material parameters. A full color version of this figure is available at Polymer Journal online.

Because this is a simple system that does not require the implementation of an algorithm to calculate a single optimal value, it could be used widely for nonlinear programming, including complex physical property values (as explained later) as well as for compositional design, including time-series data.

This system is based on linear programming; thus, the property can be roughly predicted, although it may lack precision. To increase the precision, the relational equation between the composition content and the property should preferably be expressed by a nonlinear simultaneous equation. However, in the case in which several combinations of materials must be investigated, an extremely large amount of experimental data becomes necessary to calculate the function of $n$ variables for each combination. In practice, according to the procedure shown in Figure 11, the candidate materials are narrowed down by linear approximation, and the nonlinear approximation is conducted only when precision is required.

This system was used for investigating the adhesive agent composition when several materials were combined. This system was applied for developing not only various die-bonding films, shown in Figure 2, but also for thermally conductive adhesive film, among other materials. It was found that wide ranges of functional extensions and applications, such as extension to nonlinear programming, calculation of material supply risk and database building, were possible. 


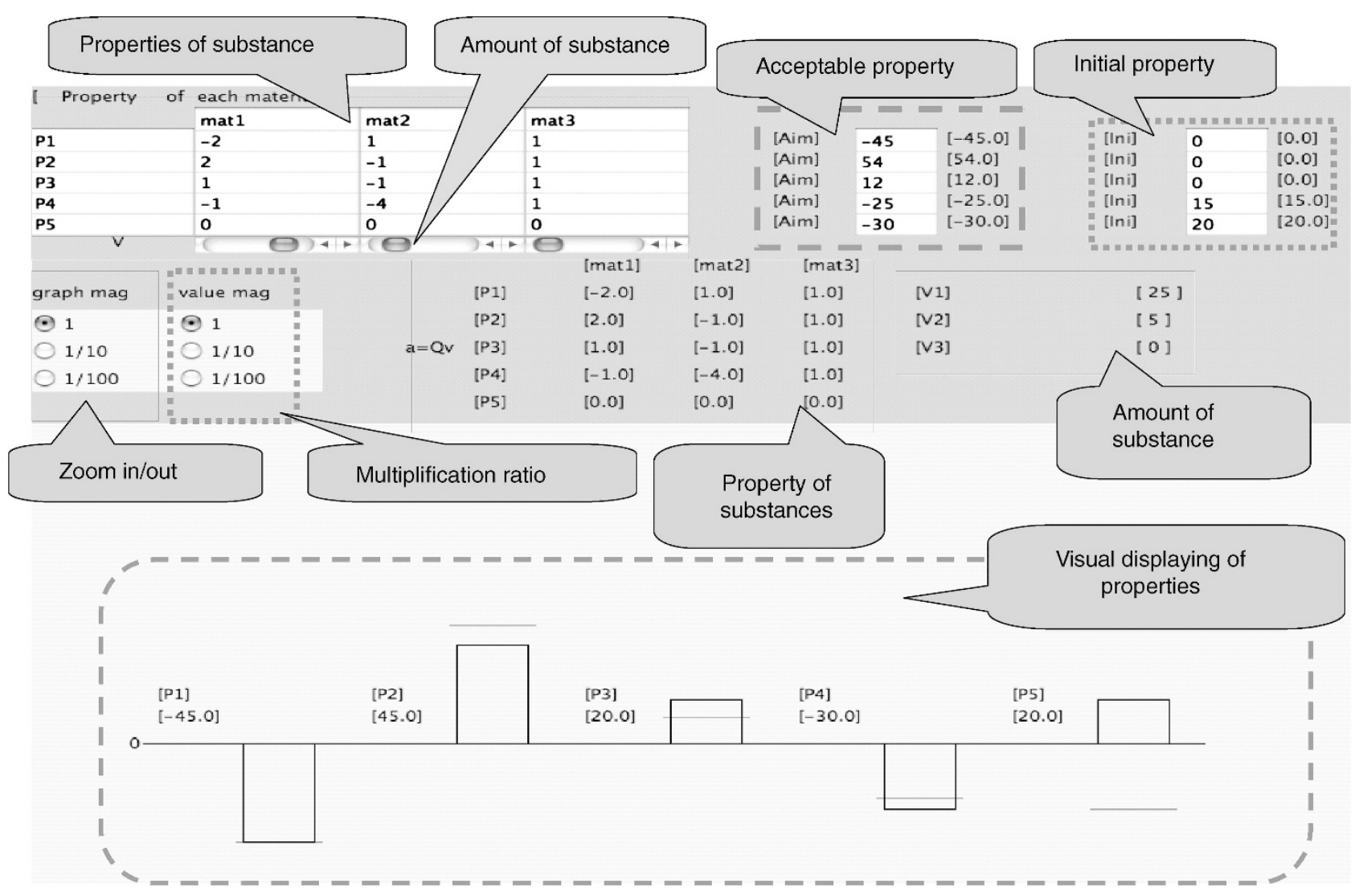

Figure 10 Example of the M-Designer Viewer. A full color version of this figure is available at Polymer Journal online.

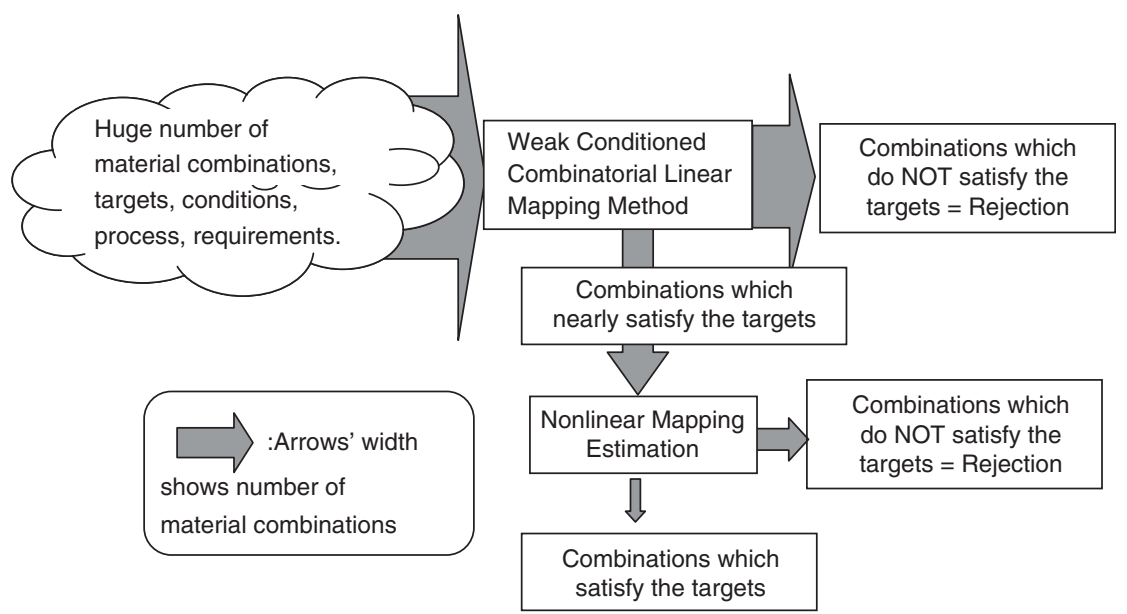

Figure 11 Flowchart of the optimization process using the weak conditioned combinatorial linear programming method and nonlinear estimation. A full color version of this figure is available at Polymer Journal online.

\section{CONCLUSIONS}

A novel die-bonding film developed by reaction-induced polymer alloy technology and material design technology shows excellent adhesion strength, reliability and heat resistance. The film also satisfied the demands of the semiconductor package assembly process. This die-bonding film is used as a de facto standard for the stacked package. The film is indispensable in the latest portable electronic devices, and it is not an overstatement to say that it is partially responsible for the explosive expansion of electronic device innovation.

Moreover, a wide range of applications are expected for the developed adhesives, which have shown natural stress relaxation and heat resistance, including automotive, alternative energy and medical applications.

A novel material design method (the weak conditioned combinatorial linear programming method) was also developed. By applying 
this method, many properties can be changed and controlled simultaneously, which saves time when adjusting the properties of die-bonding film for many type of packaging applications. This method is effective for identifying a well-balanced material that has value not only in semiconductor-related materials but also in other formulations such as paint, medicine and food. However, a single, optimal value for accuracy cannot be achieved.

\section{ACKNOWLEDGEMENTS}

Part of this research was sponsored by the New Energy and Industrial Technology Development (NEDO: Ministry of Economy, Trade and Industry, Japan) in terms of the Project on Nanostructured Polymeric Materials, launched in 2001.

1 Yamanaka, K. \& Inoue, T. Structure development in Epoxy resin modified with poly (ether sulfone). Polymer 30, 662-667 (1989).
2 Inoue, T. Reaction-induced phase decomposition in polymer blends. Prog. Polymer Sci. 20, 119-153 (1995).

3 Iwakura, T., Inada, T., Kader, M. \& Inoue, T. Structure, properties of acrylic polymer/ epoxy adhesive by reaction-induced phase decomposition. E-J. Soft Mater. 2, 13-19 (2006).

4 Inada, T., Hatakeyama, K. \& Matsuzaki, T. Reaction-induced phase separation of epoxy resin/acrylic polymer alloy and its application to die-bonding film. Netw. Polymer 25 13-19 (2004).

5 Miyauchi, K., Iwakura, T. \& Inoue, T. Control of the morphology and property of acrylic rubber/epoxy resin adhesive film by reaction-induced phase decomposition. J. Netw. Polymer, Japan 28, 48-55 (2007).

6 Inada, T. Research on the Design and Practical Use of Die-Bonding Film Using Reaction Induced Phase Separation, Doctoral Dissertation (Yamagata University, 2007).

7 Gou, Y., Aoyama, Y., Takahara, A., Jinnai, H \& Inoue, T. Evolution of high peel strength via cavitation in acrylic rubber/epoxy resin adhesive by reaction-induced phase decomposition. J. Netw. Polymer, Japan 29, 31-37 (2008).

8 Dantzig, G. Linear programming and extensions (Princeton University Press, 1963).

9 Inada, T. Research on the computational near-optimal set analysis for the programmed material development, Doctoral Dissertation (Yamagata University, 2012).

10 Inada, T. \& Matsuo, T. Property optimization of adhesive by weak conditioned combinatorial linear programming method. International Symposium on Applied Informatics, International Institute of Applied Informatics (Yonezawa, Japan, 2011).

11 Inada, T. \& Matsuo, T. Intelligent material design system based on weak conditioned linear programming. Int. J. Multimedia Ubiquitous Eng. 7 (4), 45-58 (2012).

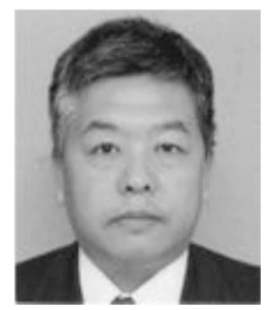

Dr Teiichi Inada was born in Tokyo in 1964. He received his B.S. degree in 1988 and master degree in 1990 from Tokyo Institute of Technology. In the same year, he joined Hitachi Chemical Co. Ltd. and started R\&D of adhesives for electric devices. He is currently a chief researcher of Tsukuba Research Laboratory. He got Ph.D. in material science at Yamagata University in 2007, also he got Ph.D. in Informatics at Yamagata University in 2012. He is a recipient of Research Award of Japan Thermosetting Plastics Industry Association (2006), Annual Technical Development Award of Japan Institute of Electronics Packaging (2008), Excellent Research Presentation Prize, at the Annual Meeting of Japan Personal Computer Application Technology Society (2011), Prime Minister's Award at the 9th Industry-Academia-Government Collaboration Contribution Awards (2011), Best Paper Award at the International Conference on Advanced Information Technology and Sensor Application (2012), The Award of the Society of Polymer Science, Japan (2012). His current research interests include design and development of electronics packaging materials and mathematical approach for the material development. 